\title{
Topological phases and fractional excitations of exciton condensate in a special class of bilayer systems
}

\author{
Ningning Hao, ${ }^{1,2}$ Ping Zhang, $, 2,3$, 田 and Yupeng Wang ${ }^{1}$ \\ ${ }^{1}$ Institute of Physics, Chinese Academy of Sciences, Beijing 100190, P. R. China. \\ ${ }^{2}$ LCP, Institute of Applied Physics and Computational Mathematics, \\ P.O. Box 8009, Beijing 100088, People's Republic of China \\ ${ }^{3}$ Center for Applied Physics and Technology, Peking University, Beijing 100871, People's Republic of China
}

\begin{abstract}
We study the exciton condensate in zero temperature limit in a special class of electron-hole bilayer systems adjacent to insulating ferromagnetic films. With the self-consistent mean-field approximation, we find that the Rashba spin-orbit interaction in the electron and hole layers can induce the $p \pm i p$ or $p$ pairing states depending on the different magnetization of the overlapped ferromagnetic films. Correspondingly, the topologically nontrivial or trivial phases emerge. Furthermore, in the topologically nontrivial phase, the quasiparticle excitations of the $U(1)$ vortex are attached to fractional quantum numbers and obey Abelian statistics.
\end{abstract}

PACS numbers: 03.65.Vf, 73.21.Fg, 73.43.Lp

\section{INTRODUCTION}

Most phases in condensed matter physics can be well understood with Landau's phase transition theory, which characterizes states of matter in terms of local order parameters associated with spontaneous breaking of the underlying symmetries. However, the quantum Hall $(\mathrm{QH})$ state [1, 2] gives the first example of topological order [3, 4] without breaking any symmetries and can be characterized by topological quantum numbers and gapless edge states. From then on, search for possible realization of nontrivial topological phases has become an intriguing and challenging issue in basic condensed matter physics as well as technological applications. Recently, the two dimensional (2D) topological band insulators (TBI) [5-8] present new topological phases which have QH effect or quantum spin Hall (QSH) effect depending on time-reversal symmetry (TRS) broken or not. Correspondingly, The 2D TBI can be well characterized by the (Thouless, Kohmoto, Nightingale, and Nijs) TKNN number [3] for the $\mathrm{QH}$ phases and by $Z_{2}$ topological number [7] for the QSH phases. Soon after, the 3D TBIs, which are the natural generalization from 2D TBIs, were discovered in several real materials such as $\mathrm{Bi}_{1-x} \mathrm{Sb}_{x}$ alloys and $\mathrm{Be}_{2} \mathrm{Se}_{3}$-family crystals $\left.9-13\right]$.

Besides 2D and 3D TBIs, more recently, a new class of topological superconductors (TSs) has been predicted by the topological classification of the Bogoliubov-deGennes (BdG) Hamiltonians [14, 15]. Most interestingly, the topological quasiparticle excitations in TSs are Majorana Fermions with non-Abelian statistics [16, 17], which have potential applications in topological quantum computation [18]. In analogy with TS, recently, we gave a proposal to realize the topological exciton condensate (TEC) in a spin-orbit coupled electron-hole bilayer sys-

\footnotetext{
*Corresponding author. zhang-ping@iapcm.ac.cn
}

tem adjacent to two insulating ferromagnetic (FM) films [19]. Note that in our previous proposal [19], the electronhole bilayer is fabricated with semiconductors such as GaAs or InAs heterostructures, where the electron and hole have different effective mass and different type of Rashba spin-orbit coupling (namely, $k$-type in electron layer and $k^{3}$-type in hole layer). Unfortunately, these differences break the particle-hole symmetry (PHS) of the bilayer system considered in Ref. [19]. We know that the PHS is indispensable for emergence of the stably topological quasiparticle excitations, such as Majorana fermion in TSs 20, 21]. Hence, we can predict no stable topological quasiparticle excitations exist in TEC proposed in Ref. [19]. In this paper, we extend the work of Ref. [19] by considering the presence of PHS and discuss the topological quasiparticle excitations.

We start with an electron-hole bilayer system shown in Fig. 1(a). In order to preserve the PHS, it is not suitable for the electron and hole layers to be fabricated with semiconductor heterostructures due to the aforementioned reasons. We argue that the recently predicted topological crystalline insulator (TCI) 22] films are appropriate candidates which have metallic surface states with quadratic dispersion on high-symmetry crystal surfaces. The robustness of the surface states of TCI are protected by the crystal point group symmetries [for example four-fold $\left(C_{4}\right)$ or six-fold $\left(C_{6}\right)$ rotational symmetry] associated with TRS of spinless fermion. Hence, the TCIs are the counterpart of $Z_{2}$ topological insulators without spin-orbit coupling. Correspondingly, when TRS of spinless fermion is broken, the TCIs are the counterpart of TKNN topological insulators. When the suitable parameters are chosen in the TCI model, the dispersion of the surface states can be described as $\epsilon_{k}= \pm k^{2} / 2 m_{\text {eff }}$. Then the electron and hole layers can be obtained by independently modulating the gate voltages attached to the TCI films. Generally, only the orbital degrees of freedom are considered in TCIs. However, the spin degrees of freedom have to be considered if the magnetic effect 


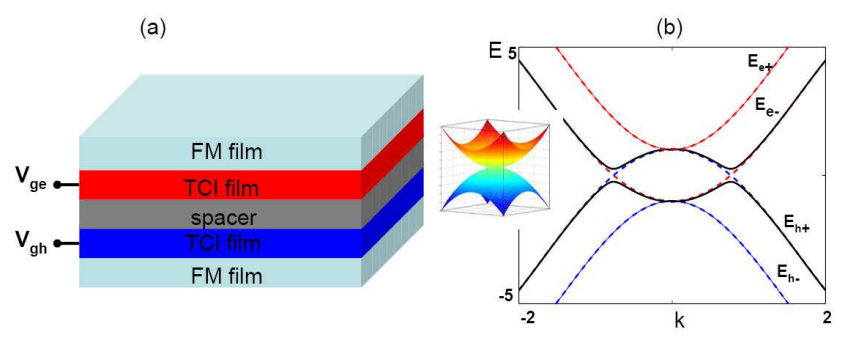

FIG. 1: (Color online) (a) Schematic structure of the effective electron-hole bilayer system that holds the exciton condensate. Different layers are fabricated with corresponding material shown on the layers, in which the spacer are fabricated with dielectric and its thickness is $d$. The electron and hole layers are obtained by tuning the gate voltages $V_{g e}$ and $V_{g h}$. The inserted picture is the dispersion of the surface state of TCI. The FM films provide effective exchange fields $\left(V_{e}, V_{h}\right)$. (b) The energy spectrum of the effective electron-hole bilayer system near the Fermi energy $E_{F}=0$. Here the dashed lines denote non-interacting single-particle energy spectrum $E_{e(h) \pm}$, while the solid lines denote the exciton energy spectrum with an obvious mean-field gap opened. We take $t_{e}=t_{h}=1, \mu_{e}=\mu_{h}=-4,\left(V_{e}, V_{h}\right)=(-1,-1)$, and $\alpha=0.5$.

is introduced, which is the case in our model. We introduce two insulating FM films adjacent to the two TCI films to support two effective interface exchange fields, which respectively induce the population imbalance of the spin-up and spin-down carriers in the electron and hole layers and break the spinful TRS of the system due to the Zeeman effect of the exchange fields. We argue that the magnetic vector potential (orbital effect) of the exchange fields of the FM films can be neglected, because the exchange fields only locate on the interface of the FM films and TCI films. That is the reason why we do not apply external magnetic fields to replace the FM films. Additionally, we assume that the exchange fields are always perpendicular to the bilayer. It is predictable that the interlayer Coulomb interaction induces the excitonic instability when the two layers are separated with an insulating spacer and close enough with each other. Moreover, We note that since structure inversion symmetry is broken at the TCI film and the insulating spacer interface, there will be an interface Rashba spin-orbit coupling (SOC) [23, 24]: $\mathcal{H}_{R}=\alpha(\sigma \times \mathbf{k}) \cdot \hat{z}$ and $\alpha \propto\left\langle\nabla_{z} V\right\rangle$, where $V$ comes from doping, impurities, external gate voltages and so on. It is evident to find that $\alpha$ is not zero when the structure inversion symmetry is broken and can be tunable by external gate voltages. Moreover, a Dresselhaus term [25] can not be zero when the bulk inversion symmetry is broken. In the two dimensions, $\mathcal{H}_{D}=\beta\left(k_{x} \sigma_{x}-k_{y} \sigma_{y}\right)$ and $\beta \approx-\mathcal{B}(\pi / w)^{2}$, where $\mathcal{B}$ is a material-dependent parameter. In the conventional semiconductor quantum-well systems, $w$ is the width of the well with a typical value of $\sim 50 \AA$, then $\beta \sim 0.3(0.1)$ $\mathrm{eV} \AA$ in InSb (GaAs) quantum wells, which is compa- rable to or even larger than the value of $\alpha$ [26]. In this case, the Dresselhaus term can not be neglected. However, if the electron and hole layers are fabricated with TCI films, since the TCI has the bulk inversion symmetry, then the Dresselhaus term can be neglected and we can only consider the Rashba term. When the amplitude of the Rashba SOC is comparable to that of the exciton order parameter (EOP), we find that the unconventional pairing states emerge with topologically nontrivial $p+i p$ pairing states or trivial $p$ pairing states depending on the magnetization of the overlapped FM films. Furthermore, we find that the topological quasiparticle excitations of the $U(1)$ vortex profiles survive and carry fractional quantum numbers [27, 28] that obey the Abilan statistics.

The paper is organized as follows. In Sec. II a model Hamiltonian for the effective electron-hole bilayer system is introduced, and a self-consistent mean-field method is used to produce the unconventional pairing states. In Sec. III we introduce the TKNN number as the topological number to characterize topologically nontrivial pairing states and show the chiral gapless edge states. In Sec. IV we analytically study the topological quasiparticle excitation of a single $U(1)$ vortex in the low-energy limit and give the numerical results in lattice model and find both are consistent. In Sec. V we summarize our results.

\section{MODEL HAMILTONIAN AND EXCITON CONDENSATE}

Consider the effective TCI electron-hole bilayer confined in the $x-y$ plane in zero temperature limit. Then the mean-field description is appropriate even in two dimensions. For convenience of discussion, here we consider a square lattice model. Furthermore, the continuous model can be obtained from the low-energy limit of the lattice model. The square lattice Hamiltonian is $\mathcal{H}=\sum_{p}\left(\mathcal{H}_{\text {kin }}^{(p)}+\mathcal{H}_{R}^{(p)}\right)+\mathcal{H}_{\text {int }}^{(e-h)}:$

$$
\begin{aligned}
\mathcal{H}_{k i n}^{(p)} & =\sum_{<i, j>, \sigma}\left(-t_{p}-\mu_{p} \delta_{i j}\right) \hat{p}_{i, \sigma}^{\dagger} \hat{p}_{j, \sigma} \\
& +\sum_{j, \sigma, \sigma^{\prime}} V_{p}\left(s_{z}\right)_{\sigma, \sigma^{\prime}} \hat{p}_{j, \sigma}^{\dagger} \hat{p}_{j, \sigma^{\prime}}, \\
\mathcal{H}_{R}^{(p)} & =\frac{1}{2} \alpha_{p}\left[\sum_{j}\left(\hat{p}_{j, \uparrow}^{\dagger} \hat{p}_{j+\delta x, \downarrow}-\hat{p}_{j, \uparrow}^{\dagger} \hat{p}_{j-\delta x, \downarrow}\right)\right. \\
& \left.-i \sum_{j}\left(\hat{p}_{j, \uparrow}^{\dagger} \hat{p}_{j+\delta y, \downarrow}-\hat{p}_{j, \uparrow}^{\dagger} \hat{p}_{j-\delta y, \downarrow}\right)\right]+ \text { H.c. }, \\
\mathcal{H}_{i n t}^{(e-h)} & =-\sum_{i, j, \sigma, \sigma^{\prime}} U_{i, j}(d) \hat{e}_{i \sigma}^{\dagger} \hat{h}_{j \sigma^{\prime}}^{\dagger} \hat{h}_{j \sigma^{\prime}} \hat{e}_{i \sigma} .
\end{aligned}
$$

Here $t_{p}$ denotes the nearest-neighbor hopping amplitude while $\mu_{p}$ represents the chemical potential in electron $(p=e)$ or hole $(p=h)$ layer. Without loss of generality, we set $t_{p}=1$ for both electron and hole layer, other parameters are measured in $t_{p} . s_{x, y, z}$ are the three Pauli 
matrices and $s_{0}$ is the identity matrix. $V_{p}$ represents Zeeman splitting from the effective interface exchange field of the FM film. $\hat{p}_{j, \sigma}$ is the annihilation operator of carrier with spin $\sigma$ at lattice site $j . \quad \alpha_{e(h)}$ is the Rashba $\mathrm{SO}$ interaction strength in the electron (hole) layer. For simplicity, in this paper we set $\alpha_{e}=\alpha_{h}=\alpha . \delta x(\delta y)$ is the square-lattice spacing measured in unit of in-plane lattice constant along the $x(y)$ direction. The interlayer Coulomb interaction is $U_{i, j}(d)=e^{2} / \varepsilon \sqrt{\left|\mathbf{r}_{i, e}-\mathbf{r}_{j, h}\right|^{2}+d^{2}}$, where $\varepsilon$ is the dielectric constant of the spacer and $d$ is the interlayer distance. We only consider the interaction relevant to exciton formation and ignore the electronhole exchange interaction. We also neglect the intralayer electron-electron and hole-hole interactions, since they are expected to renormalize the single-particle spectrum of each layer and have no essential influence on the topological properties of the system.

In momentum space, the Hamiltonian Eq. (1) can be expressed as

$$
\begin{aligned}
\mathcal{H}_{k i n}^{(p)} & =\sum_{\mathbf{k}, \sigma, \sigma^{\prime}}\left[\left(\zeta_{\mathbf{k}}^{(p)}-\mu_{p}\right) \delta_{\sigma, \sigma^{\prime}}+V_{p}\left(s_{z}\right)_{\sigma, \sigma^{\prime}}\right] \hat{p}_{\mathbf{k}, \sigma}^{\dagger} \hat{p}_{\mathbf{k}, \sigma^{\prime}}, \\
\mathcal{H}_{R}^{(p)} & =\sum_{\mathbf{k}} i \alpha\left(\sin k_{x}-i \sin k_{y}\right) \hat{p}_{\mathbf{k} \uparrow}^{\dagger} \hat{p}_{\mathbf{k} \downarrow}+\text { H.c. } \\
\mathcal{H}_{i n t}^{(e-h)} & =\sum_{\mathbf{k} \mathbf{k}^{\prime} \mathbf{q} \sigma \sigma^{\prime}}-\frac{U(\mathbf{q})}{\Omega} \hat{e}_{\mathbf{k}+\mathbf{q} \sigma}^{\dagger} \hat{h}_{\mathbf{k}^{\prime}-\mathbf{q} \sigma^{\prime}}^{\dagger} \hat{h}_{\mathbf{k}^{\prime} \sigma^{\prime}} \hat{e}_{\mathbf{k} \sigma},
\end{aligned}
$$

where $U(\mathbf{q})=\frac{2 \pi e^{2}}{\varepsilon q} e^{-q d}$ and $\zeta_{\mathbf{k}}^{(p)}=-2 t_{p}\left(\cos k_{x}+\cos k_{y}\right)$. In the Nambu notation with combined $\hat{e}-\hat{h}$ field operator basis $\hat{\Psi}_{\mathbf{k}}=\left[\hat{e}_{\mathbf{k} \uparrow} \hat{e}_{\mathbf{k} \downarrow} \hat{h}_{-\mathbf{k} \uparrow}^{\dagger} \hat{h}_{-\mathbf{k} \downarrow}^{\dagger}\right]^{T}$, the decoupled mean-field Hamiltonian is expressed as $\mathcal{H}_{M F}=\hat{\Psi}^{\dagger}\left(\mathcal{H}_{0}+\mathcal{H}_{1}\right) \hat{\Psi}+E_{0}$ in the following Bogoliubov-de Gennes (BdG) form:

$\mathcal{H}_{M F}=\sum_{\mathbf{k}} \hat{\Psi}_{\mathbf{k}}^{\dagger}\left[\begin{array}{cc}\boldsymbol{\Sigma}_{\mathbf{k}}^{(e)}-\mu_{e}+V_{e} s_{z} & \boldsymbol{\Delta}(\mathbf{k}) \\ \boldsymbol{\Delta}^{\dagger}(\mathbf{k}) & \boldsymbol{\Sigma}_{-\mathbf{k}}^{(h)}+\mu_{h}-V_{h} s_{z}\end{array}\right] \hat{\Psi}_{\mathbf{k}}$,

where $\mathcal{H}_{0}$ represents the single-particle terms while $\mathcal{H}_{1}$ includes the interaction terms, $\boldsymbol{\Sigma}_{ \pm \mathbf{k}}^{(p)}= \pm \zeta_{ \pm \mathbf{k}}^{(p)} s_{0} \pm \mathcal{H}_{R}^{(p)}( \pm \mathbf{k})$, $\Delta_{\sigma \sigma^{\prime}}(\mathbf{k})=-\frac{1}{\Omega} \sum_{\mathbf{q}} U(\mathbf{q})\left\langle\hat{h}_{-\mathbf{k}+\mathbf{q} \sigma^{\prime}} \hat{e}_{\mathbf{k}-\mathbf{q} \sigma}\right\rangle$ are the EOPs, and $E_{0}=\frac{1}{\Omega} \sum_{\mathbf{k}, \mathbf{q} \sigma \sigma^{\prime}} \frac{\Delta_{\sigma \sigma^{\prime}}(\mathbf{k}) \Delta_{\sigma \sigma^{\prime}}^{*}(\mathbf{k}-\mathbf{q})}{U(\mathbf{q})}$ in which we have neglected the inessential constants.

Moreover, the exciton condensate with unconventional pairing can be well understood in the Fermi surface nesting picture. To reveal this fact, firstly, the noninteracting Hamiltonian $\mathcal{H}_{0}$ in Eq. (3) can be diagonalized with the unitary transformation in the single-particle eigenstate space as

$$
\mathcal{H}_{0}=\sum_{\mathbf{k}} \hat{\Psi}_{0 \mathbf{k}}^{\dagger}\left(H_{E}^{(e)}(\mathbf{k}) \oplus H_{E}^{(h)}(\mathbf{k})\right) \hat{\Psi}_{0 \mathbf{k}},
$$

where $H_{E}^{(p)}(\mathbf{k})=E_{p-}(\mathbf{k})\left(s_{0}+s_{z}\right) / 2+E_{p+}(\mathbf{k})\left(s_{0}-s_{z}\right) / 2$, $\hat{\Psi}_{0 \mathbf{k}}=\left[\hat{\psi}_{e+}(\mathbf{k}), \hat{\psi}_{e-}(\mathbf{k}), \hat{\psi}_{h+}^{\dagger}(\mathbf{k}), \hat{\psi}_{h-}^{\dagger}(\mathbf{k})\right]^{T}$,
$E_{p s}(k)= \pm \zeta_{ \pm \mathbf{k}}^{(p)} \mp \mu_{p}+s \sqrt{\alpha^{2}\left(\sin ^{2} k_{x}+\sin ^{2} k_{y}\right)+V_{p}^{2}}$

$(s=+,-)$ are respectively electron and hole band energies, and $\hat{\psi}_{p s}$ denote the relevant particle annihilation operators. The two basis sets are related by the corresponding unitary transformation, $\hat{\Psi}_{\mathbf{k}}=e^{i \theta_{\mathbf{k}}} \prod_{e} \oplus e^{i \vartheta_{\mathbf{k}}} \Pi_{h}^{*}$ $\hat{\Psi}_{0 \mathbf{k}}$ with

$$
\Pi_{p}=\left[\begin{array}{cc}
-i f_{p+}(k) e^{-i \phi_{\mathbf{k}}} & f_{p-}(k) \\
f_{p-}(k) & -i f_{p+}(k) e^{i \phi_{\mathbf{k}}}
\end{array}\right]
$$

where

and

$f_{p \pm}(\mathbf{k})=\omega_{\mathbf{k}} / \sqrt{\omega_{\mathbf{k}}^{2}+\left(\sqrt{\omega_{\mathbf{k}}^{2}+V_{p}^{2}} \pm V_{p}\right)^{2}} \quad$ with

$\omega_{\mathbf{k}}=\alpha \sqrt{\sin ^{2} k_{x}+\sin ^{2} k_{y}}$. It should be stressed that the $k$-dependent phases $\theta_{k}$ and $\vartheta_{k}$ are confirmed by exactly solving the ground state of the system through self-consistent mean-field calculation. The single-particle bands $E_{p \pm}(\mathbf{k})$ are shown in Fig. 1(b) (dashed curves), in which the occupied bands are $E_{e-}$ and $E_{h+}$. With the values of the tunable parameters shown in the caption in Fig. 1 , the band $E_{e-}$ and band $E_{h+}$ have the perfect identical Fermi surface. From the nesting mechanism, the attractive interlayer interaction leads to unstability of the Fermi surfaces and opens a gap. This is the case similar to the conventional BCS picture. Now, the electron-hole interaction part relevant to exciton formation in Eq. (2) can be expressed in terms of the occupied electron band $E_{e-}$ and hole band $E_{h+}$ with Eq. (5). Then, the decoupled mean-field two-band Hamiltonian of the exciton system under the basis $\bar{\Psi}=\left[\hat{\psi}_{e-}(\mathbf{k}), \hat{\psi}_{h+}^{\dagger}(-\mathbf{k})\right]^{T}$ reads (with inessential constants neglected)

$$
\overline{\mathcal{H}}_{M F}=\sum_{\mathbf{k}} \bar{\Psi}_{\mathbf{k}}^{\dagger}\left[\begin{array}{cc}
E_{e-} & \bar{\Delta}(\mathbf{k}) \\
\bar{\Delta}^{*}(\mathbf{k}) & E_{h+}
\end{array}\right] \bar{\Psi}_{\mathbf{k}}+\bar{E}_{0},
$$

where $\bar{\Delta}(\mathbf{k})=-\frac{1}{\Omega} \sum_{\mathbf{q}, s, s^{\prime}} U_{r e}\left(\mathbf{k}, \mathbf{q}, s, s^{\prime}\right)\left\langle\psi_{h+}(-\mathbf{k}+\mathbf{q}) \psi_{e-}(\mathbf{k}-\mathbf{q})\right\rangle$ and $\quad \bar{E}_{0}=\frac{1}{\Omega} \sum_{\mathbf{k q}} \frac{\bar{\Delta}(\mathbf{k}) \bar{\Delta}^{*}\left(\mathbf{k}^{\prime}\right)}{\sum_{s s^{\prime}} U_{r e}^{*}\left(\mathbf{k}, \mathbf{q}, s, s^{\prime}\right)} \quad$ with renormalized interaction $\quad U_{r e}\left(\mathbf{k}, \mathbf{q}, s, s^{\prime}\right)=U(\mathbf{q}) \chi_{\mathbf{k}, \mathbf{q}} F_{s s^{\prime}}(\mathbf{k}, \mathbf{q}) \tau_{\mathbf{k}, \mathbf{q}}$, $s= \pm, \quad \chi_{\mathbf{k}, \mathbf{q}}=e^{i\left(\theta_{\mathbf{k}-\mathbf{q}}-\theta_{\mathbf{k}}\right)} e^{i\left(\vartheta_{\mathbf{k}}-\vartheta_{\mathbf{k}-\mathbf{q}}\right)}, \quad$ and $F_{s s^{\prime}}(\mathbf{k}, \mathbf{q})=f_{e s}(k) f_{e s}(k-q) f_{e s^{\prime}}(k) f_{e s^{\prime}}(k-q) . \quad$ Here, $\tau_{\mathbf{k}, \mathbf{q}}=e^{i\left(\phi_{\mathbf{k}-\mathbf{q}}-\phi_{\mathbf{k}}\right)}$ for $s \neq s^{\prime}, \quad \tau_{\mathbf{k}, \mathbf{q}}=1$ for $s=s^{\prime}=-$, and $\tau_{\mathbf{k}, \mathbf{q}}=e^{i 2\left(\phi_{\mathbf{k}-\mathbf{q}}-\phi_{\mathbf{k}}\right)}$ for $s=s^{\prime}=+$. Then the EOPs in Eq. (3) and those in Eq. (6) are related by the unitary transformation associated with Eq. (5), i.e.,

$$
\begin{aligned}
\boldsymbol{\Delta}(\mathbf{k}) & =-e^{i\left(\theta_{\mathbf{k}}-\vartheta_{\mathbf{k}}\right)} \bar{\Delta}(\mathbf{k}) \\
& \times\left[\begin{array}{cc}
i f_{e+}(\mathbf{k}) f_{h+} e^{-i \phi_{\mathbf{k}}} & f_{e+}(\mathbf{k}) f_{h-}(\mathbf{k}) \\
-f_{e-}(\mathbf{k}) f_{h+}(\mathbf{k}) & i f_{e-}(\mathbf{k}) f_{h-}(\mathbf{k}) e^{i \phi_{\mathbf{k}}}
\end{array}\right] .
\end{aligned}
$$

The factors $f_{e \pm}(\mathbf{k}) f_{h \pm}(\mathbf{k})$ in Eq. (7) can be understood as follows. Since the four pairing states with different 


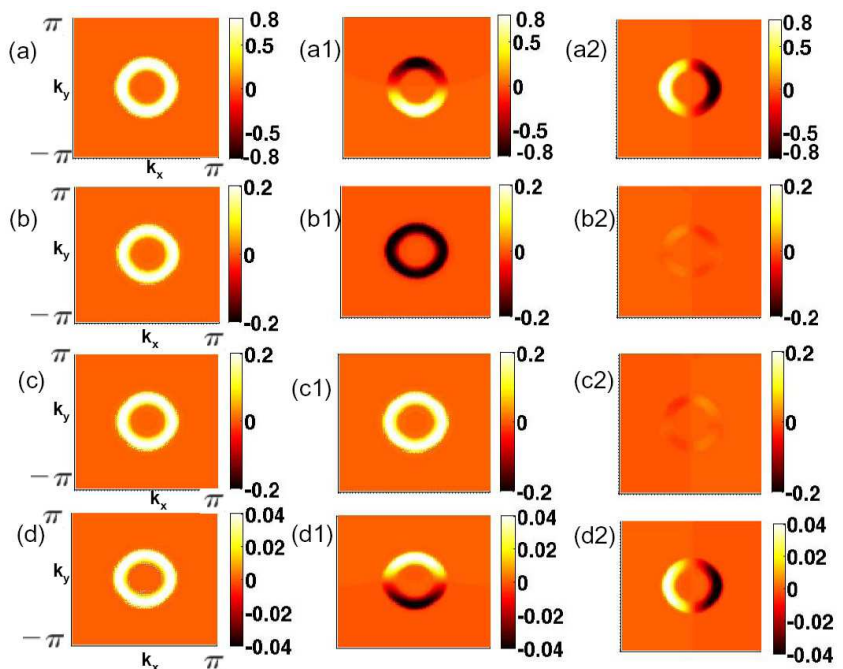

FIG. 2: (Color online) The self-consistent results of EOPs versus momentum $\left(k_{x}, k_{y}\right)$. (a), (b), (c) and (d) refer to $\left|\Delta_{\uparrow \uparrow}(\mathbf{k})\right|$, $\left|\Delta_{\uparrow \uparrow}(\mathbf{k})\right|,\left|\Delta_{\downarrow \uparrow}(\mathbf{k})\right|$ and $\left|\Delta_{\downarrow \downarrow}(\mathbf{k})\right|$. Correspondingly, (a1), (b1), (c1) and (d1) refer to $\operatorname{Re}\left(\Delta_{\uparrow \uparrow}(\mathbf{k})\right), \operatorname{Re}\left(\Delta_{\uparrow \uparrow}(\mathbf{k})\right), \operatorname{Re}\left(\Delta_{\downarrow \uparrow}(\mathbf{k})\right)$ and $\operatorname{Re}\left(\Delta_{\downarrow \downarrow}(\mathbf{k})\right)$; (a2), (b2), (c2) and (d2) refer to $\operatorname{Im}\left(\Delta_{\uparrow \uparrow}(\mathbf{k})\right)$, $\operatorname{Im}\left(\Delta_{\uparrow \uparrow}(\mathbf{k})\right), \operatorname{Im}\left(\Delta_{\downarrow \uparrow}(\mathbf{k})\right)$ and $\operatorname{Im}\left(\Delta_{\downarrow \downarrow}(\mathbf{k})\right)$. The effective Zeeman fields are $\left(V_{e}, V_{h}\right)=(-1,-1)$.
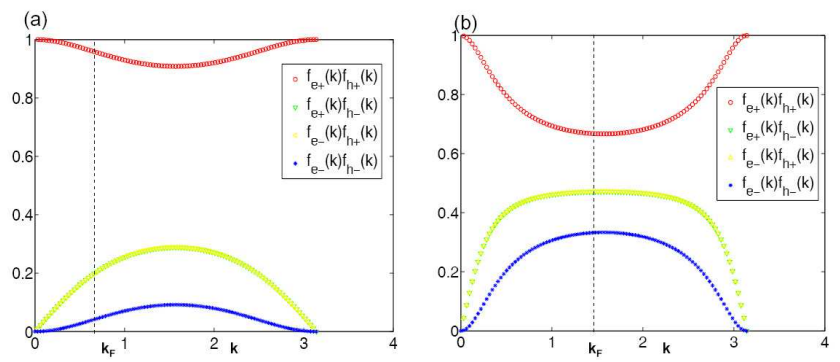

FIG. 3: (Color online) The coefficients $f_{e+}(\mathbf{k}) f_{h+}(\mathbf{k})$, $f_{e+}(\mathbf{k}) f_{h-}(\mathbf{k}), \quad f_{e-}(\mathbf{k}) f_{h+}(\mathbf{k})$ and $f_{e-}(\mathbf{k}) f_{h-}(\mathbf{k})$ versus the momentum $k$ in the magnetization configuration $\left(V_{e}, V_{h}\right)=(-1,-1)$ for different Rashba SOC values: (a) $\alpha=0.5$; (b) $\alpha=2$.

spin combinations in EOPs are affected differently by the effective exchange fields. Additionally, the Rashba SOC flaws the spin polarization of the carries along the $z$ direction, then the total effect leads the factors $f_{e \pm}(\mathbf{k}) f_{h \pm}(\mathbf{k})$ to emerge in four pairing states of EOPs and determine the EOP behavior at given $\left(V_{e}, V_{h},\right)$. We find that only one component of $\Delta_{\sigma \sigma^{\prime}}(\mathbf{k})$ is dominant, while the other components can be neglected in the proper range of the Rashba SOC parameter for the given magnetization of the FM films. Moreover, the system's properties are decided by the dominant component of $\Delta_{\sigma \sigma^{\prime}}(\mathbf{k})$. For definiteness, we have carried out a series of selfconsistent numerical solutions of the BdG equation (3) by setting the lattice size $81 \times 81, \mu_{e}=\mu_{h}=-4, \alpha=0.5$, and
$\left(V_{e}, V_{h}\right)=( \pm 1, \pm 1)$. As a typical example, we show in Fig. 2 the numerical results of $\Delta_{\sigma \sigma^{\prime}}(\mathbf{k})$ for $\left(V_{e}, V_{h}\right)=(-1,-1)$. Also, we present in Fig. 3(a) the results of the factors $f_{e \pm}(\mathbf{k}) f_{h \pm}(\mathbf{k})$ for comparison. In this case, from Fig. 2 it is clear that $\Delta_{\uparrow \uparrow}(\mathbf{k})$ is the dominant EOP component which has the phase of $-i e^{-i \phi_{\mathbf{k}}}$, i.e. $e^{i\left(\theta_{\mathbf{k}}-\vartheta_{\mathbf{k}}\right)}=1.0$. In the continuous limit, $-i e^{-i \phi_{\mathbf{k}}} \sim-\frac{k_{y}+i k_{x}}{k}$, which means the $p+i p$-like pairing state. Furthermore, this kind of pairing state is usually topologically nontrivial (see discussion below). Similarly, for the case of $\left(V_{e}, V_{h}\right)=(1,1)$, the $\Delta_{\downarrow \downarrow}(\mathbf{k})$ turns out to be the dominant pairing component with a phase of $-i e^{i \phi_{\mathbf{k}}}$, which thus is also topologically nontrivial. On the other hand, for the case of $\left(V_{e}, V_{h}\right)=(-1,1)$, the $\Delta_{\uparrow \downarrow}(\mathbf{k})$ is the dominant pairing component with a phase of $-\left(e^{i \phi_{\mathbf{k}}}+e^{-i \phi_{\mathbf{k}}}\right)$. The physics related to this kind of phase is that $e^{i\left(\theta_{\mathbf{k}}-\vartheta_{\mathbf{k}}\right)}=e^{ \pm i \phi_{\mathbf{k}}}$ correspond to two degenerate states and their linear combinations give the ground state with the phase of $-\left(e^{i \phi_{\mathbf{k}}}+e^{-i \phi_{\mathbf{k}}}\right)$. In the continuum limit, $-\left(e^{i \phi_{\mathbf{k}}}+e^{-i \phi_{\mathbf{k}}}\right) \sim-\frac{k_{x}}{k}$. This kind of pairing state is topologically trivial. Similarly, for the case of $\left(V_{e}, V_{h}\right)=(1,-1)$, the dominant pairing component $\Delta_{\downarrow \uparrow}(\mathbf{k})$ is also topologically trivial.

Furthermore, from Fig. 3 (a) and Eq. (7) we can find that the weak Rashba SOC relative to the Zeeman fields will select one dominating component of EOPs (namely, $\Delta_{\uparrow \uparrow}(\mathbf{k})$ ), while other components can be neglected. But in Fig. 3 (b) we can find that the amplitudes of $f_{e+}(k) f_{h+}(k), f_{e+}(k) f_{h-}(k)$ and $f_{e-}(k) f_{h+}(k)$ are comparable. That means the non-weak Rashba SOC relative to the exchange field cannot select one dominating component of EOPs, and all components of EOPs play important roles. The consequence is that the unconventional pairing states become unstable in case of Fig. 3 (b), where the value of $\alpha$ is chosen to be much larger than the exchange filed. We can also clarify it from numerical calculation. Hence, the Rashba SOC cannot be larger than the Zeeman splitting in our system. Fortunately, it is easy to satisfy this requirement, because both Rashba SOC and Zeeman fields are tunable in our system. Besides, another essential point we stress is that the topologically trivial or nontrivial EC in our system is stable, which means a stable bulk gap separating the ground state and the excited states. We can obviously find this fact in Fig. 1(b) and Fig. 4(a) (see below). In the following section, we focus on the characterization of the topological properties of the system.

\section{CHIRAL TOPOLOGICAL ORDER}

From the self-consistent results of the aforementioned section, we can find that the EOPs are closely correlated to the magnetization configuration of the exchange fields. We also note that there are two different classes: $\left(V_{e}, V_{h}\right)=( \pm 1, \pm 1)$ and $\left(V_{e}, V_{h}\right)=( \pm 1, \mp 1)$. So we choose one configuration from each class as an example. The EOPs can be simplified as $\Delta_{\uparrow \uparrow}(\mathbf{k})=-i \Delta_{0}\left(\sin k_{x}-\right.$ $\left.i \sin k_{y}\right)$ and other $\Delta_{\sigma \sigma^{\prime}}(\mathbf{k}) \sim 0$ for $\left(V_{e}, V_{h}\right)=(-1,-1)$, 
while $\Delta_{\uparrow \downarrow}(\mathbf{k})=-\Delta_{0} \sin k_{x}$ and other $\Delta_{\sigma \sigma^{\prime}}(\mathbf{k}) \sim 0$ for $\left(V_{e}, V_{h}\right)=(-1,1)$. Then the mean-field Hamiltonian Eq. (3) in these two magnetization configurations can be adiabatically mapped into the following forms, which are topologically equivalent to initial mean-field Hamiltonian Eq. (3) when $\alpha \rightarrow 0$ without the bulk gap closing [29].

$$
\begin{gathered}
\mathcal{H}_{M F}^{t}=\sum_{\mathbf{k}}\left[\hat{e}_{\mathbf{k} \uparrow}, \hat{h}_{-\mathbf{k} \uparrow}^{\dagger}\right] H^{t}(\mathbf{k})\left[\begin{array}{c}
\hat{e}_{\mathbf{k} \uparrow}^{\dagger} \\
\hat{h}_{-\mathbf{k} \uparrow}
\end{array}\right] \\
\mathcal{H}_{M F}^{s}=\sum_{\mathbf{k}}\left[\hat{e}_{\mathbf{k} \uparrow}, \hat{h}_{-\mathbf{k} \downarrow}^{\dagger}\right] H^{s}(\mathbf{k})\left[\begin{array}{c}
\hat{e}_{\mathbf{k} \uparrow}^{\dagger} \\
\hat{h}_{-\mathbf{k} \downarrow}
\end{array}\right]
\end{gathered}
$$

where $H^{t}(\mathbf{k})=\left(\zeta_{\mathbf{k}}^{(e)}-\mu_{e}+V_{e}\right)\left(s_{0}+s_{3}\right) / 2+\left(-\zeta_{\mathbf{k}}^{(h)}+\mu_{h}-\right.$ $\left.V_{h}\right)\left(s_{0}-s_{3}\right) / 2-i \Delta_{0}\left(\sin k_{x} s_{1}+\sin k_{y} s_{2}\right)$ and $H^{s}(\mathbf{k})=$ $\left(\zeta_{\mathbf{k}}^{(e)}-\mu_{e}+V_{e}\right)\left(s_{0}+s_{3}\right) / 2+\left(-\zeta_{\mathbf{k}}^{(h)}+\mu_{h}+V_{h}\right)\left(s_{0}-\right.$ $\left.s_{3}\right) / 2-\Delta_{0} \sin k_{x} s_{1}$. It is easy to check that both $H^{s}(\mathbf{k})$ and $H^{t}(\mathbf{k})$ preserve the PHS and break the TRS according to the definition of Ref. [30]. The missing of TRS can be traced back to the effective Zeeman fields in Eq. (3). From Ref. [30], we can find that the Hamiltonian describing our system generally belongs to class $D$ in BdG class. The topologically nontrivial pairing has the form of chiral $p$ wave (namely, $p_{x}+i p_{y}$ or $p_{x}-i p_{y}$ pairing) in two dimensions. The $p_{x}$ or $p_{y}$ pairing is trivial. Furthermore, the nontrivial topology is characterized by an integer which is similar to the TKNN number in the QHE.

We can calculate this topological number from Eq. (3). The topological nature of the ground state of the exciton condensate can be characterized by non-zero $I_{T K N N}$, which reads

$$
I_{T K N N}=-\frac{1}{2 \pi} \sum_{n=1}^{2} \int_{B Z} d^{2} k \Omega_{n}(\mathbf{k}),
$$

where $\Omega_{n}(\mathbf{k})=-2 \operatorname{Im}\left\langle\frac{\partial u_{n}(\mathbf{k})}{\partial k_{x}} \mid \frac{\partial u_{n}(\mathbf{k})}{\partial k_{y}}\right\rangle$ is the Berry curvature of the occupied energy band $n$ and $\left|u_{n}(\mathbf{k})\right\rangle$ is the corresponding Bloch wave function. The straightforward calculation gives $I_{T K N N}=1$ for $\left(V_{e}, V_{h}\right)=(-1,-1)$ and $I_{T K N N}=0$ for $\left(V_{e}, V_{h}\right)=(-1,1)$. From the bulk-edge correspondence, the nontrivial bulk topological number implies gapless edge states emerging in the terminal of the system.

In order to evaluate gapless edge states, we assume that the square-lattice system has two edges in $y$ direction and is boundless in $x$ direction. Correspondingly, we choose open boundary condition in $y$ direction and periodic boundary condition in $x$ direction in the lattice Hamiltonian in Eq. (10) in mean-field approximation. The calculated energy spectrums of the topologically nontrivial cases of $\left(V_{e}, V_{h}\right)=(-1,-1)$ and topologically trivial case of $\left(V_{e}, V_{h}\right)=(-1,1)$ are illustrated in Fig. 4 (a) and (b), respectively, in which the red-solid and blue-dashed lines correspond to the different edge states with opposite chiralities. It is obvious to find that (a)

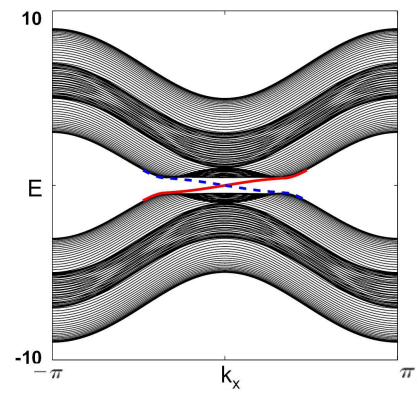

(b)

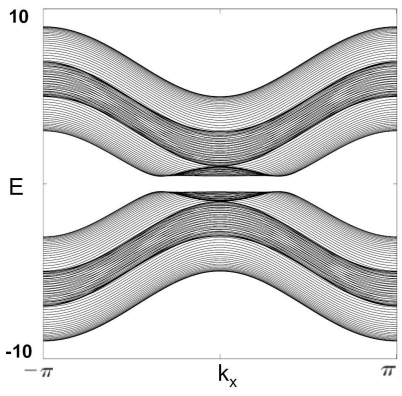

FIG. 4: (Color online) The energy spectrum of the bilayer square-lattice system with two edges at the $y$ direction. $k_{x}$ denotes the momentum in the $x$ direction. The magnetization parameters are set at $\left(V_{e}, V_{h}\right)=(-1,-1)$ for (a) and $\left(V_{e}, V_{h}\right)=(-1,1)$ for (b). In both cases $\Delta_{0}=0.5$ and $\alpha=0.5$. The red-solid and blue-dashed lines denote the edge states locating at different edges.

the number of the gapless edge states is consistent with the bulk theory characterized by $I_{T K N N}$.

Furthermore, for the case of $\left(V_{e}, V_{h}\right)=(-1,-1)$, the edge states can be described by the effective Hamiltonian:

$$
H_{\text {edge }}= \pm \sum_{k_{x} \geq 0} v_{F} k_{x} \hat{\gamma}^{\dagger}\left(k_{x}, y\right) \hat{\gamma}\left(k_{x}, y\right)
$$

where \pm represents the opposite chiralities, $v_{F}$ is the Fermi velocity, and $k_{x}$ is the momentum measured from the Fermi surface, and

$$
\hat{\gamma}\left(k_{x}, y\right)=u\left(k_{x}, y\right) \hat{e}_{\uparrow}(y)+v\left(k_{x}, y\right) \hat{h}_{\uparrow}^{\dagger}(y) .
$$

We will show in next section that the low-energy topological quasiparticle excitations are dominated by the edge states.

\section{FRACTIONAL QUSIPARTICLE EXCITATIONS IN EXCITON CONDENSATE}

We now demonstrate that there exist fractional excitations in our system in the topologically nontrivial phases if the EOPs have a $U(1)$ vortex profile with odd vorticity. Note that the even vorticity is trivial [31]. For definiteness, we consider the case: $\left(V_{e}, V_{h}\right)=(-V,-V)=(-1,-1)$ and $\mu_{e}=\mu_{h}=\mu=-4 t_{p}$. The non-vortex BdG Hamiltonian in Eq. (3) reads

$$
\mathcal{H}_{M F}(\mathbf{k})=\left[\begin{array}{cc}
\boldsymbol{\Sigma}_{\mathbf{k}}^{(e)}-\mu-V s_{z} & \boldsymbol{\Delta}(\mathbf{k}) \\
\boldsymbol{\Delta}^{\dagger}(\mathbf{k}) & \boldsymbol{\Sigma}_{-\mathbf{k}}^{(h)}+\mu+V s_{z}
\end{array}\right]
$$

with 


$$
\boldsymbol{\Delta}(\mathbf{k})=\left[\begin{array}{cc}
-i \Delta_{0}\left(\sin k_{x}-i \sin k_{y}\right) & 0 \\
0 & 0
\end{array}\right]
$$

The PHS of $\mathcal{H}_{M F}(\mathbf{k})$ is defined by $\Lambda \mathcal{H}_{M F}(\mathbf{k}) \Lambda=-\mathcal{H}_{M F}(-\mathbf{k})$ with $\Lambda=s_{x} \otimes s_{0} \mathcal{K}$, where $\mathcal{K}$ is the complex-conjugation operator and $\Lambda^{2}=1$.

Now, we consider a vortex in the EOPs, i.e., the uniform $\Delta_{0}$ in Eq. (14) is modulated to $f^{2}(r) e^{i n \theta}$, where $r$ and $\theta$ are polar coordinates centered on the vortex, $n$ is the vorticity, and $f(r)$ is a real function of $r$ that vanishes at small $r$ and obtains asymptotic value $f_{0}$ (a real constant) at large $r$. Usually, the nature of the excitation bound to the vortex is dominated by the low-energy limit of the system, i.e. $\mathbf{k} \rightarrow 0$. In this limit, the effective Hamiltonian can be obtained by the replacement $\mathbf{k} \rightarrow-i \nabla$ and is accurate up to $\mathcal{O}\left(\mathbf{k}^{2}\right)$. The zero-mode equation reads $\mathcal{H}_{M F}(-i \nabla) \Psi_{\mathbf{0}}=0$ with $\Psi_{\mathbf{0}}(r, \theta)=\left[u_{\uparrow}, u_{\downarrow}, v_{\uparrow}, v_{\downarrow}\right]^{T}$. With the help of $\Lambda \mathcal{H}_{M F}(-i \nabla) \Lambda=-\mathcal{H}_{M F}(-i \nabla)$, it is easy to find $v_{\sigma}=u_{\sigma}^{*}$. Hence the zero-mode equations can be expressed as
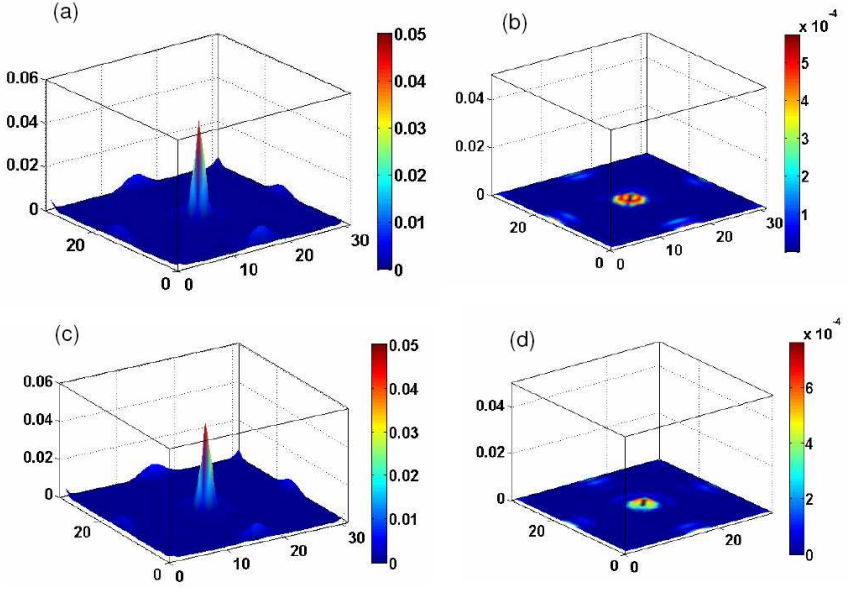

FIG. 5: (Color online) The density distributions of quasiparticle excitation of the $U(1)$ vortex locating on the center of a $31 \times 31$ lattice for (a) $\left|u_{\uparrow}(r)\right|^{2}$, (b) $\left|u_{\downarrow}(r)\right|^{2}$, (c) $\left|v_{\uparrow}(r)\right|^{2}$, and (d) $\left|v_{\downarrow}(r)\right|^{2}$ in the exchange fields $\left(V_{e}, V_{h}\right)=(-1,-1)$. We take $f_{0}^{2}=0.5, \alpha=0.5$.

the vortex zero mode in topological superconductor is $\hat{\gamma}_{s c}=\int d \mathbf{r}\left(u_{\uparrow} \hat{c}_{\uparrow}(\mathbf{r})+v_{\uparrow} \hat{c}_{\uparrow}^{\dagger}(\mathbf{r})\right)[21,32]$. It is obvious to find that $\hat{\gamma}_{e c} \neq \hat{\gamma}_{e c}^{\dagger}$ due to $\left[\hat{e}_{\uparrow}(\mathbf{r}), \hat{h}_{\uparrow}^{\dagger}(\mathbf{r})\right]_{+}=0$ and $\hat{\gamma}_{s c}=\hat{\gamma}_{s c}^{\dagger}$ due $-\alpha \frac{\partial u_{\uparrow}}{\partial z}+\left(-\frac{\partial^{2}}{\partial z \partial z^{*}}+V\right) u_{\downarrow}=0$,

where $z=x+i y$. From Eq. (12) in Sec. IV, we can find that $u_{\downarrow}$ can be neglected. Moreover, the numerical results shown in Fig. 5 also give a proof. Hence, we only need to consider Eq. (15). For definiteness we consider the simplest case in the quantum limit analogous to the London approximation in superconductor, i.e., $f(r=0)=0$, $f(r>0)=f_{0}$ (a positive real constant) and the $U(1)$ vortex with vorticity $n=1$. Moreover, we look for a solution in terms of a spherically symmetric real function $u_{\uparrow}$. Note that in Eq. (16), we must take into account the $U(1)$ symmetry in order to get the physical solutions. Then Eq. (15) can be simplified into the following differential equation:

$$
\frac{d^{2} u_{\uparrow}}{d r^{2}}+\left(\frac{1}{r}+f_{0}^{2}\right) \frac{d u_{\uparrow}}{d r}+\left(\frac{f_{0}^{2}}{2 r}+V\right) u_{\uparrow}=0 .
$$

The solution of Eq. (17) is $u_{\uparrow} \propto$ $J_{0}\left(\sqrt{4 V^{2}+f_{0}^{4}} r / 2\right) e^{-f_{0}^{2} r / 2}$ where $J_{0}(x)$ is the Bessel function. We also give the exact numerical results of $\left|u_{\sigma}\right|^{2}$ and $\left|v_{\sigma}\right|^{2}$ in the square-lattice system, which are shown in Fig. 5. Clearly, it can be seen that $u_{\downarrow} \sim 0$, which is consistent with Eq. (12).

The quasiparticle excitation field associated with the zero-mode solution in the exciton condensate can be expressed as $\hat{\gamma}_{e c}=\int d \mathbf{r}\left(u_{\uparrow} \hat{e}_{\uparrow}(\mathbf{r})+v_{\uparrow} \hat{h}_{\uparrow}^{\dagger}(\mathbf{r})\right)$. Contrastively, the quasiparticle excitation field describing to $\left[\hat{c}_{\uparrow}(\mathbf{r}), \hat{c}_{\uparrow}^{\dagger}(\mathbf{r})\right]_{+}=1$. That means the quasiparticle excitations in topological superconductor are the Majorana fermions which obey the non-Abelian anyonic statistics 17]. In our case, the quasiparticle excitations are not the Majorana fermions and are charged. Generally, a localized zero mode in a system with PHS is known to carry a fractional charge of $\pm e / 2$ 27, 33]. Based on the arguments given in Refs. 34, 35], we expect for the present system that the quasiparticle excitation bounding to vortice has fractional charge $e / 2$. We can also evaluate it from the spatial integration $\int d \mathbf{r}\left(\left|u_{\uparrow}\right|^{2}+\left|v_{\uparrow}\right|^{2}\right)$ around the vortex defect. Fractional charged quasiparticles confined to 2D often obey fractional exchange statistics [36], which is in our case Abelian from the standard arguments [37.

\section{CONCLUSION}

In conclusion, we have studied the exciton condensate in zero temperature limit in a special class of effective TCI bilayer system which is different from the semiconductor bilayer system in Ref. [19] due to its preservation of PHS. With the self-consistent mean-field method, we have found that the Rashba SOC in the electron and hole layers can induce the unconventional $p \pm i p$ or $p$ pairing states that depend on the magnetization of the FM insulating films. Correspondingly, the $p \pm i p$ pairing states represent topologically nontrivial exciton condensate phase, which can be characterized by nonzero TKNN number or the gapless edge states, while the $p$ par- 
ing states are topologically trivial. Furthermore, by lowenergy analytic solution as well as exact numerical calculation, we have shown that in the topologically nontrivial exciton condensate phase, the presence of $U(1)$ vortexes result in the simple Fermi zero-mode fractional quasiparticle excitations that obey Abelian statistics, which completely distinguish from the non-Abelian Majorana Fermi zero-mode quasiparticle excitations in topological superconductor systems.

\section{Acknowledgments}

This work was supported by NSFC under Grants No. 90921003, No. 10574150, and No. 60776063, and by the National Basic Research Program of China (973 Program) under Grants No. 2009CB929103 and No. 2011 CB921701.
[1] K. V. Klitzing, G. Dorda and M. Pepper, Phys. Rev. Lett. 45, 494 (1980).

[2] D. C. Tsui, H. L. Stormer and A. C. Gossard, Phys. Rev. Lett. 48, 1559 (1982).

[3] D. J. Thouless, M. Kohmoto, M. P. Nightingale and M. den Nijs, Phys. Rev. Lett. 49, 405 (1982)

[4] X. G.Wen, Advances in Physics 44, 405 (1995).

[5] F. D. M. Haldane, Phys. Rev. Lett. 61, 2015 (1988).

[6] C. L. Kane and E. J. Mele, Phys. Rev. Lett. 95, 226801 (2005).

[7] C. L. Kane and E. J. Mele, Phys. Rev. Lett. 95, 146802 (2005).

[8] B. A. Bernevig, T. A. Hughes, and S. C. Zhang, Science 314, 1757 (2006).

[9] L. Fu and C. L. Kane, Phys. Rev. B 76, 045302 (2007).

[10] D. Hsieh, D. Qian, L. Wray, Y. Xia, Y. S. Hor, R. J. Cava, and M. Z. Hasan, Nature 452, 970 (2008).

[11] H. J. Zhang, C. X. Liu, X. L. Qi, X. Dai, Z. Fang, and S. C. Zhang, Nature Phys. 5, 438 (2009).

[12] Y. Xia, D. Qian, D. Hsieh, L. Wrayl, A. Pal1, H. Lin, A. Bansil, D. Grauer, Y. S. Hor, R. J. Cava, Nat. Phys. 5, 398 (2009).

[13] Y. L. Chen, J. G. Analytis, J. H. Chu, Z. K. Liu, S. K. Mo, X. L. Qi, H. J. Zhang, D. H. Lu, X. Dai, Z. Fang, Science 325, 178 (2009).

[14] A. Schynder, S. Ryu, A. Furusaki and A. Ludwig, Phys. Rev. B 78, 195125 (2008).

[15] A. Kitaev, arXiv: 0901.2686.

[16] D. A. Ivanov, Phys. Rev. Lett., 86, 268 (2001).

[17] N. Read and D. Green Phys. Rev. B, 61, 10267 (2000).

[18] C. Nayak, S. H. Simon, A. Stern, M. Freedman, and S. D. Sarma, Rev. Mod. Phys 80, 1083 (2008).
[19] N. Hao, P. Zhang, J. Li, Z. Wang, W. Zhang and Y. Wang, Phys. Rev. B, 82, 195324 (2010).

[20] Jay D. Sau, Roman M. Lutchyn, Sumanta Tewari, and S. Das Sarma, Phys. Rev. Lett. 104, 040502 (2010).

[21] M. Sato, Y. Takahashi, and S. Fujimoto, Phys. Rev. Lett. 103, 020401 (2009).

[22] L. Fu, Phys. Rev. Lett. 106, 106802 (2011).

[23] E. I. Rashba. Sov. Phys. Solid State, 2, 1109 (1960).

[24] Yu. A. Bychkov and E. I. Rashba. JETP Lett. 39, 78 (1984).

[25] G. Dresselhaus, Phys. Rev. 100, 580 (1955).

[26] J. Alicea, Phys. Rev. B 81, 125318 (2010).

[27] R. Jackiw and C. Rebbi, Phys. Rev. D 13, 3398 (1976).

[28] W. P. Su, J. R. Schrieffer, and A. J. Heeger, Phys. Rev. Lett. 42, 1698 (1979).

[29] M. Sato and S. Fujimoto, Phys. Rev. B 79, 094504 (2009).

[30] A. P. Schnyder, S. Ryu, A. Furusaki and A. W. W. Ludwig, Phys. Rev. B 78, 195125 (2008).

[31] V. Gurarie and L. Radzihovsky, Phys. Rev. B 75, 212509 (2008).

[32] X. Qi, T. L. Hughes, S. Raghu, and S. Zhang, Phys. Rev. Lett. 102, 187001 (2009).

[33] J. Goldstone and F. Wilczek, Phys. Rev. Lett. 47, 986 (1981).

[34] I. F. Herbut, Phys. Rev. Lett. 99, 206404 (2007).

[35] B. Seradjeh, J. E. Moore, and M. Franz, Phys. Rev. Lett. 103, 066402 (2009)

[36] R. B. Laughlin, Phys. Rev. Lett. 50, 1395 (1983).

[37] F. Wilczek, Fractional Statistics and Anyon Superconductivity (World Scientific, Singapore, 1990). 